\title{
Construction of Choquet integrals through unimodal weighting vectors
}

\author{
Bonifacio Llamazares* \\ Departamento de Economía Aplicada, Instituto de Matemáticas (IMUVA), \\ and BORDA Research Unit, Universidad de Valladolid, 47011 Valladolid, Spain
}

\begin{abstract}
Semiuninorm-based ordered weighted averaging (SUOWA) operators are a specific case of Choquet integrals that allow us to generalize simultaneously weighted means and ordered weighting averaging (OWA) operators. Although SUOWA operators possess some very interesting properties, their main weakness is that, sometimes, the game used in their construction is not monotonic and it is necessary to calculate its monotonic cover. In this paper, we introduce a new family of weighting vectors, called unimodal weighting vectors, which embrace some of the most outstanding weighting vectors used in the framework of OWA operators, and we show that when using these weighting vectors and a specific semiuninorm we directly get normalized capacities. Moreover, we also show that these operators satisfy some properties which are very useful in practice.
\end{abstract}

\section{INTRODUCTION}

The study of aggregation operators is a hot topic in several scientific fields that is generating a rich literature. ${ }^{1-3}$ An interesting issue is the construction of functions that allow us to combine weighted means and ordered weighting averaging (OWA) operators ${ }^{4}$ in a single function. Remember that both weighted means and OWA operators are defined by means of nonnegative vectors that sum to one (weighting vectors). However, the role of the weighting vectors in both families of functions is quite different: in the weighted means, the weights allow us to take into account the importance of the information sources while in the OWA operators the weights allows us to take into account the relative size of the values.

The usual approach followed in the literature for combining weighted means and OWA operators is to consider functions parametrized by two weighting vectors, $\boldsymbol{p}$ for the weighted mean and $\boldsymbol{w}$ for the OWA operator, so that we can recover the weighted mean when $\boldsymbol{w}=(1 / n, \ldots, 1 / n)$ and the OWA operator when $\boldsymbol{p}=(1 / n, \ldots, 1 / n)$. Among the solutions proposed in the literature, ${ }^{5}$

*e-mail: boni@eco.uva.es. 
the weighted OWA (WOWA) operators, ${ }^{6}$ and the semiuninorm-based ordered weighted averaging (SUOWA) operators ${ }^{7}$ are very interesting because they can be expressed through Choquet integrals with respect to normalized capacities (it is worth noting thatBeliakov ${ }^{8}$ has suggested recently a new method of introducing weights into an OWA type aggregation). In this way, both families of functions are monotonic, compensative, idempotent, continuous, and homogeneous of degree 1.

The study of SUOWA operators has been carried out in several papers, ${ }^{7,9-13}$ where it has been shown that these functions exhibit some interesting properties. Nevertheless, the main weakness of these functions is that, sometimes, the construction of the capacities is not straightforward given that it is necessary to calculate the monotonic cover of certain games, which are obtained by using semiuninorms ${ }^{14}$ with neutral element $1 / n$ and the values of the capacities associated with the weighted means and the OWA operators.

The aim of this paper is to show that the previous weakness can be avoided on numerous occasions. For that, we introduce a new family of weighting vectors, called unimodal weighting vectors, that includes some of the most outstanding weighting vectors used in the literature of OWA operators: nonincreasing, nondecreasing, centered weighting vectors, etc. Through the use of these weighting vectors and a specific semiuninorm, $U_{\min }^{\max }$, we show that the games used in the construction of SUOWA operators are normalized capacities, which are explicitly given in the paper. Of particular interest is the case where the OWA operator has a behavior similar to that of the winsorized means; that is, where it is the arithmetic mean of the values after replacing the highest and/or lowest values with the most extreme remaining value(s). In this case, the SUOWA operator is the weighted mean of the values after applying the same procedure; that is, after replacing the highest and/or lowest values with the most extreme remaining value(s).

We also study some properties of these operators. Specifically, we prove that the weight affecting the component $x_{i}$ is located between the corresponding weights of the weighted mean and the OWA operator. Likewise, we analyze these capacities in relation to the balancing, subadditivity and superadditivity properties and we also show that their dual capacities are obtained by using the dual of the weighting vectors $\boldsymbol{w}$. Lastly, we emphasize that these operators preserve the conjunctive/disjuntive character of the OWA operator associated with them, so that we get functions located between two order statistics that take into account the weights of the information sources.

The remainder of the paper is organized as follows. In Section 2 we present a brief survey of Choquet integral, including weighted means and OWA operators. Section 3 is devoted to recall the definition and basic properties of semiuninorms and SUOWA operators. In Section 4 we introduce unimodal weighting vectors and show that a lot of weighting vectors used in the literature of OWA operators are unimodal. Sections 5 and 6 collect the main results of the paper: In Section 5 we establish the results that allow us to construct the capacities through unimodal weighting vectors, whereas in Section 6 we analyze some properties of 
these capacities. Finally, some concluding remarks are provided in Section 7.

\section{CHOQUET INTEGRAL}

The following notation will be used throughout the paper: $N=\{1, \ldots, n\}$; given $A \subseteq N, A^{\mathrm{c}}$ and $|A|$ denote, respectively, the complement and the cardinality of $A$; vectors are denoted in bold; $\boldsymbol{\eta}$ denotes the tuple $(1 / n, \ldots, 1 / n) \in \mathbb{R}^{n}$; and we will write $\boldsymbol{x} \geq \boldsymbol{y}$ if $x_{i} \geq y_{i}$ for all $i \in N$. Given $\boldsymbol{x} \in \mathbb{R}^{n}$, [.] and (.) denote permutations such that $x_{[1]} \geq \cdots \geq x_{[n]}$ and $x_{(1)} \leq \cdots \leq x_{(n)}$. Moreover, given $a \in \mathbb{R},\lfloor a\rfloor$ and $\lceil a\rceil$ will denote, respectively, the floor and the ceiling of $a$; i.e., the largest integer smaller than or equal to $a$, and the smallest integer larger than or equal to $a$.

Given $F: \mathbb{R}^{n} \longrightarrow \mathbb{R}$, some well-known properties of $F$ are the following:

1. Symmetry: $F\left(x_{\sigma(1)}, \ldots, x_{\sigma(n)}\right)=F\left(x_{1}, \ldots, x_{n}\right)$ for all $\boldsymbol{x} \in \mathbb{R}^{n}$ and for all permutation $\sigma$ of $N$.

2. Monotonicity: $\boldsymbol{x} \geq \boldsymbol{y}$ implies $F(\boldsymbol{x}) \geq F(\boldsymbol{y})$ for all $\boldsymbol{x}, \boldsymbol{y} \in \mathbb{R}^{n}$.

3. Idempotency: $F(x, \ldots, x)=x$ for all $x \in \mathbb{R}$.

4. Compensativeness (or internality): $\min (\boldsymbol{x}) \leq F(\boldsymbol{x}) \leq \max (\boldsymbol{x})$ for all $\boldsymbol{x} \in$ $\mathbb{R}^{n}$.

5. Homogeneity of degree 1 (or ratio scale invariance): $F(r \boldsymbol{x})=r F(\boldsymbol{x})$ for all $\boldsymbol{x} \in \mathbb{R}^{n}$ and $r>0$.

In the last years, Choquet integral ${ }^{15}$ has become more and more important in the field of decision theory, due mainly to its simplicity, versatility and good properties. Choquet integrals are defined from capacities ${ }^{15}$ (also known in the literature as fuzzy measures ${ }^{16}$ ). The notion of capacity is similar to that of probability measure, where the additivity property is changed by monotonicity. And a game is a generalization of a capacity without the monotonicity assumption.

\section{DEFINITION 1.}

1. A game $v$ on $N$ is a set function, $v: 2^{N} \longrightarrow \mathbb{R}$ satisfying $v(\varnothing)=0$.

2. A capacity (or fuzzy measure) $\mu$ on $N$ is a monotonic game on $N$ (that is, $\mu(A) \leq \mu(B)$ whenever $A \subseteq B)$. In particular, it follows that $\mu: 2^{N} \longrightarrow$ $[0, \infty)$. A capacity $\mu$ is said to be normalized if $\mu(N)=1$.

Given a game $v$, the monotonic cover ${ }^{17,18}$ of $v$ is the smallest capacity that contains it.

Definition 2. Let $v$ be a game on $N$. The monotonic cover of $v$ is the set function $\hat{v}$ given by

$$
\hat{v}(A)=\max _{B \subseteq A} v(B) .
$$


By construction, $\hat{v}$ is a capacity on $N$ and, when $v$ is a capacity, $\hat{v}=v$. Moreover, the monotonic cover of a game satisfies the following property.

Remark 1. Let $v$ be a game on $N$. If $v(A) \leq 1$ for all $A \subseteq N$ and $v(N)=1$, then $\hat{v}$ is a normalized capacity.

The dual of a normalized capacity is defined as follows.

Definition 3. Let $\mu$ be a normalized capacity on $N$.

1. The dual capacity of $\mu$, denoted as $\bar{\mu}$, is the normalized capacity defined by

$$
\bar{\mu}(A)=1-\mu\left(A^{\mathrm{c}}\right) \quad(A \subseteq N) .
$$

2. $\mu$ is self-dual if $\bar{\mu}=\mu$.

Although the Choquet integral is usually defined as a functional, ${ }^{15,19}$ in the discrete case it can also be seen as an aggregation function over $\mathbb{R}^{n}$ (see, for instance, Grabisch et al. ${ }^{2}$, p. 181). Moreover, by similarity with the definition of OWA operators, we represent it by using nonincreasing sequences of values. ${ }^{7,20}$

Definition 4. Let $\mu$ be a capacity on $N$. The Choquet integral with respect to $\mu$ is the function $\mathcal{C}_{\mu}: \mathbb{R}^{n} \longrightarrow \mathbb{R}$ given by

$$
\mathcal{C}_{\mu}(\boldsymbol{x})=\sum_{i=1}^{n} \mu\left(A_{[i]}\right)\left(x_{[i]}-x_{[i+1]}\right),
$$

where $A_{[i]}=\{[1], \ldots,[i]\}$, and we use the convention $x_{[n+1]}=0$.

Choquet integral can also be written by explicitly showing the weights of the components $x_{[i]}$,

$$
\mathcal{C}_{\mu}(\boldsymbol{x})=\sum_{i=1}^{n}\left(\mu\left(A_{[i]}\right)-\mu\left(A_{[i-1]}\right)\right) x_{[i]},
$$

where we use the convention $A_{[0]}=\varnothing$. Some useful properties of the Choquet integral are given in the following remarks (see, for instance, Grabisch et al. ${ }^{2}$, pp. 192-196).

Remark 2. If $\mu$ is a normalized capacity on $N$, then $\mathcal{C}_{\mu}$ is continuous, monotonic, idempotent, compensative and homogeneous of degree 1.

Remark 3. Let $\mu_{1}$ and $\mu_{2}$ be two capacities on $N$. Then $\mu_{1} \leq \mu_{2}$ if and only if $\mathcal{C}_{\mu_{1}} \leq \mathcal{C}_{\mu_{2}}$.

Two of the most popular specific cases of Choquet integral are the weighted means and the OWA operators. ${ }^{4}$ Both are defined by using weight distributions that add up to 1 .

Definition 5. A vector $\boldsymbol{q} \in[0,1]^{n}$ is a weighting vector if $\sum_{i=1}^{n} q_{i}=1$.

Definition 6. Let $\boldsymbol{p}$ and $\boldsymbol{w}$ be two weighting vectors. 
1. The weighted mean associated with $\boldsymbol{p}$ is the function $M_{\boldsymbol{p}}: \mathbb{R}^{n} \longrightarrow \mathbb{R}$ given by

$$
M_{\boldsymbol{p}}(\boldsymbol{x})=\sum_{i=1}^{n} p_{i} x_{i} .
$$

2. The OWA operator associated with $\boldsymbol{w}$ is the function $O_{\boldsymbol{w}}: \mathbb{R}^{n} \longrightarrow \mathbb{R}$ given by

$$
O_{\boldsymbol{w}}(\boldsymbol{x})=\sum_{i=1}^{n} w_{i} x_{[i]} .
$$

The normalized capacities from which we obtain weighted means and OWA operators are given in the following remark. ${ }^{7,21-23}$

Remark 4. Let $\boldsymbol{p}$ and $\boldsymbol{w}$ be two weighting vectors.

1. The weighted mean $M_{\boldsymbol{p}}$ is the Choquet integral with respect to the normalized capacity $\mu_{\boldsymbol{p}}(A)=\sum_{i \in A} p_{i}$.

2. The OWA operator $O_{\boldsymbol{w}}$ is the Choquet integral with respect to the normalized capacity $\mu_{|\boldsymbol{w}|}(A)=\sum_{i=1}^{|A|} w_{i}$.

So, according to Remark 2, weighted means and OWA operators are continuous, monotonic, idempotent, compensative and homogeneous of degree 1. Moreover, OWA operators are also symmetric given that the values of the variables are previously ordered in a nonincreasing way.

It is also well known that $\mu_{\boldsymbol{p}}$ is self-dual; that is, $\bar{\mu}_{\boldsymbol{p}}=\mu_{\boldsymbol{p}}$, and that $\bar{\mu}_{|\boldsymbol{w}|}=\mu_{|\overline{\boldsymbol{w}}|}$, where $\overline{\boldsymbol{w}}$ is the dual of $\boldsymbol{w}$; that is, the weighting vector defined by $\bar{w}_{i}=w_{n+1-i}$, $i=1, \ldots, n$.

\section{SUOWA OPERATORS}

SUOWA operators ${ }^{7}$ were introduced for dealing with situations where both the importance of information sources and the importance of values have to be taken into account. They are defined through semiuninorms, ${ }^{14}$ which are monotonic functions with a neutral element in the interval $[0,1]$. Semiuninorms were suggested as a generalization of uninorms ${ }^{24}$ which in turn were introduced as a generalization of t-norms and t-conorms.

Definition 7. A function $U:[0,1]^{2} \longrightarrow[0,1]$ is a semiuninorm if it is monotonic and has a neutral element $e \in[0,1](U(e, x)=U(x, e)=x$ for all $x \in[0,1])$. A semiuninorm $U$ is a uninorm if it is symmetric and associative $(U(x, U(y, z))=U(U(x, y), z)$ for all $x, y, z \in[0,1])$.

The set of semiuninorms (respectively, idempotent semiuninorms) with neutral element $e \in[0,1]$ will be denoted by $\mathcal{U}^{e}$ (respectively, $\mathcal{U}_{\mathrm{i}}^{e}$ ).

The semiuninorms employed in the definition of SUOWA operators have $1 / n$ as neutral element and have to belong to the following subset ${ }^{7}$ :

$$
\widetilde{\mathcal{U}}^{1 / n}=\left\{U \in \mathcal{U}^{1 / n} \mid U(1 / k, 1 / k) \leq 1 / k \text { for all } k \in N\right\} .
$$




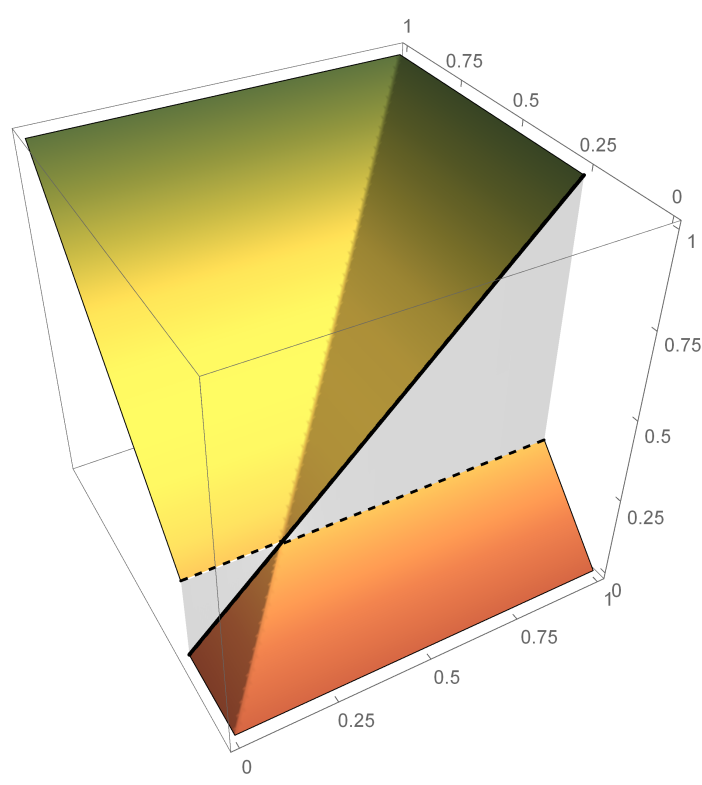

Figure 1: The semiuninorm $U_{\min }^{\max }$ for $n=4$.

Obviously $\mathcal{U}_{\mathrm{i}}^{1 / n} \subseteq \widetilde{\mathcal{U}}^{1 / n}$. Note also that the smallest and the largest elements of $\mathcal{U}_{\mathrm{i}}^{1 / n}$ are, respectively, the following uninorms ${ }^{24}$ :

$$
U_{\min }(x, y)= \begin{cases}\max (x, y) & \text { if }(x, y) \in[1 / n, 1]^{2} \\ \min (x, y) & \text { otherwise }\end{cases}
$$

and

$$
U_{\max }(x, y)= \begin{cases}\min (x, y) & \text { if }(x, y) \in[0,1 / n]^{2} \\ \max (x, y) & \text { otherwise. }\end{cases}
$$

In addition to $U_{\min }$ and $U_{\max }$, another interesting idempotent semiuninorm, which is used in this paper, is the following ${ }^{10}$ :

$$
U_{\min }^{\max }(x, y)= \begin{cases}\min (x, y) & \text { if } y<1 / n \\ x & \text { if } y=1 / n \\ \max (x, y) & \text { if } y>1 / n\end{cases}
$$

The plot of this semiuninorm, for the case $n=4$, is given in Figure 1 .

SUOWA operators a specific case of the Choquet integral where their capacities are the monotonic cover of certain games. These games are constructed through semiuninorms with neutral element $1 / n$ and the values of the capacities associated with the weighted means and the OWA operators. Specifically, the definition of these games is the following.

DeFinition 8. Let $\boldsymbol{p}$ and $\boldsymbol{w}$ be two weighting vectors and let $U \in \widetilde{\mathcal{U}}^{1 / n}$. 
1. The game associated with $\boldsymbol{p}, \boldsymbol{w}$ and $U$ is the set function $v_{\boldsymbol{p}, \boldsymbol{w}}^{U}: 2^{N} \longrightarrow \mathbb{R}$ defined by

$$
v_{\boldsymbol{p}, \boldsymbol{w}}^{U}(A)=|A| U\left(\frac{\mu_{\boldsymbol{p}}(A)}{|A|}, \frac{\mu_{|\boldsymbol{w}|}(A)}{|A|}\right)
$$

if $A \neq \varnothing$, and $v_{\boldsymbol{p}, \boldsymbol{w}}^{U}(\varnothing)=0$.

2. $\hat{v}_{\boldsymbol{p}, \boldsymbol{w}}^{U}$, the monotonic cover of the game $v_{\boldsymbol{p}, \boldsymbol{w}}^{U}$, will be called the capacity associated with $\boldsymbol{p}, \boldsymbol{w}$ and $U$.

Notice that $v_{\boldsymbol{p}, \boldsymbol{w}}^{U}(A) \leq 1$ for all $A \subseteq N$ and $v_{\boldsymbol{p}, \boldsymbol{w}}^{U}(N)=1$. Therefore, according to Remark $1, \hat{v}_{\boldsymbol{p}, \boldsymbol{w}}^{U}$ is always a normalized capacity.

DeFinition 9. Let $\boldsymbol{p}$ and $\boldsymbol{w}$ be two weighting vectors and let $U \in \widetilde{\mathcal{U}}^{1 / n}$. The SUOWA operator associated with $\boldsymbol{p}, \boldsymbol{w}$ and $U$ is the function $S_{\boldsymbol{p}, \boldsymbol{w}}^{U}: \mathbb{R}^{n} \longrightarrow \mathbb{R}$ given by

$$
S_{\boldsymbol{p}, \boldsymbol{w}}^{U}(\boldsymbol{x})=\sum_{i=1}^{n} s_{i} x_{[i]},
$$

where $s_{i}=\hat{v}_{\boldsymbol{p}, \boldsymbol{w}}^{U}\left(A_{[i]}\right)-\hat{v}_{\boldsymbol{p}, \boldsymbol{w}}^{U}\left(A_{[i-1]}\right)$ for all $i \in N, \hat{v}_{\boldsymbol{p}, \boldsymbol{w}}^{U}$ is the capacity associated with $\boldsymbol{p}, \boldsymbol{w}$ and $U$, and $A_{[i]}=\{[1], \ldots,[i]\}$ (with the convention that $A_{[0]}=\varnothing$ ).

According to expression (1), the SUOWA operator associated with $\boldsymbol{p}, \boldsymbol{w}$ and $U$ can also be written as

$$
S_{\boldsymbol{p}, \boldsymbol{w}}^{U}(\boldsymbol{x})=\sum_{i=1}^{n} \hat{v}_{\boldsymbol{p}, \boldsymbol{w}}^{U}\left(A_{[i]}\right)\left(x_{[i]}-x_{[i+1]}\right) .
$$

By the construction of $\hat{v}_{\boldsymbol{p}, \boldsymbol{w}}^{U}$, we have $S_{\boldsymbol{p}, \boldsymbol{\eta}}^{U}=M_{\boldsymbol{p}}$ and $S_{\boldsymbol{\eta}, \boldsymbol{w}}^{U}=O_{\boldsymbol{w}}$ for any $U \in \widetilde{\mathcal{U}}^{1 / n}$. Moreover, by Remark 2 and since $\hat{v}_{\boldsymbol{p}, \boldsymbol{w}}^{U}$ is a normalized capacity, SUOWA operators are continuous, monotonic, idempotent, compensative and homogeneous of degree 1 .

\section{UNIMODAL WEIGHTING VECTORS}

A fundamental issue when using an OWA operator is the choice of the weighting vector. So, this topic has generated an abundant literature in the last years, ${ }^{25-27}$ which has motivated the emergence of numerous families of OWA operators. Two of the most outstanding are the window-OWA operators ${ }^{28}$ (also known as trimmed means when they are self-dual) and the centered OWA operators. ${ }^{29}$

DeFinition 10. A window-OWA operator is an OWA operator defined by means of a weighting vector of the form

$$
w_{i}= \begin{cases}1 / m & \text { if } i=r, \ldots, r+m-1 \\ 0 & \text { otherwise }\end{cases}
$$

where $r, m \in N$, with $r+m \leq n+1$. 
Note that window-OWA operators allow us to remove extreme values in the aggregation process and, in this way, to avoid the bias caused by extreme inputs. Some well-known specific cases of window-OWA operators are the arithmetic mean, the median, the olympic OWA operators ${ }^{30}$ (where $w_{1}=w_{n}=0$ and $w_{2}=\cdots=w_{n-1}=1 /(n-2)$ ) and the order statistics (also known as Step-OWA operators $\left.{ }^{28}\right)$ : The $k$ th order statistic $\operatorname{OS}_{k}(\boldsymbol{x})=x_{(k)}$ is obtained when $w_{n-k+1}=1$, and the remaining weights are equal to zero.

DEFINITION 11. An OWA operator is said to be a centered OWA operator if its associated weighting vector $\boldsymbol{w}$ satisfies the following conditions:

1. $w_{i}=w_{n+1-i}, \quad i=1, \ldots,\lfloor n / 2\rfloor$.

2. $w_{i}<w_{j}$ whenever $i<j \leq\lfloor(n+1) / 2\rfloor$, or $i>j \geq\lfloor(n+1) / 2\rfloor$.

3. $w_{1}>0$.

Notice that centered OWA operators allow to give more importance to the central values and less weight to the extreme scores. Specific cases of centered OWA operators are the following ones:

1. Binomial OWA operators:

$$
w_{i}=\frac{\left(\begin{array}{c}
n-1 \\
i-1
\end{array}\right)}{2^{n-1}}, \quad i=1, \ldots, n
$$

2. Gaussian OWA operators ${ }^{29,31}$ :

$$
w_{i}=\frac{\mathrm{e}^{-\left(i-\mu_{n}\right)^{2} /\left(2 \sigma_{n}^{2}\right)}}{\sum_{j=1}^{n} \mathrm{e}^{-\left(j-\mu_{n}\right)^{2} /\left(2 \sigma_{n}^{2}\right)}}, \quad i=1, \ldots, n,
$$

where $\mu_{n}=(n+1) / 2$ and $\sigma_{n}^{2}=\left(n^{2}-1\right) / 12$.

On the basis of unimodal sequences of real numbers (a well-known concept in the literature of sequences; see, for instance, Brenti ${ }^{32}$ and references therein), we introduce a new family of OWA operators that generalizes the previous ones.

\section{DEFINITION 12.}

1. A finite sequence of real numbers $a_{1}, a_{2}, \ldots, a_{n}$ is unimodal if there exists an index $k$ such that $a_{1} \leq \cdots \leq a_{k-1} \leq a_{k} \geq a_{k+1} \geq \cdots \geq a_{n}$.

2. An OWA operator is said to be unimodal if its associated weighting vector $\boldsymbol{w}$ is unimodal.

Besides centered and window-OWA operators, the following families of OWA operators are also unimodal:

1. OWA operators associated with nonincreasing weighting vectors $\left(w_{1} \geq\right.$ $\left.w_{2} \geq \cdots \geq w_{n}\right)$. For instance: 
(a) Orlike-window OWA operators ${ }^{28}$ :

$$
w_{i}= \begin{cases}1 / m & \text { if } i=1, \ldots, m \\ 0 & \text { otherwise }\end{cases}
$$

(b) Orlike S-OWA operators ${ }^{33}$ :

$$
w_{i}= \begin{cases}\lambda+\frac{1-\lambda}{n} & \text { if } i=1 \\ \frac{1-\lambda}{n} & \text { otherwise }\end{cases}
$$

where $\lambda \in[0,1]$.

(c) Optimistic exponential OWA operators ${ }^{34}$ :

$$
w_{i}= \begin{cases}\alpha(1-\alpha)^{i-1} & \text { if } i=1, \ldots, n-1 \\ (1-\alpha)^{n-1} & \text { otherwise }\end{cases}
$$

where $\alpha \in[0.5,1]$.

(d) Binomial OWA operators introduced by Calvo and De Baets ${ }^{35}$ :

$$
w_{i}=\frac{\left(\begin{array}{c}
n-i \\
j-1
\end{array}\right)}{\left(\begin{array}{l}
n \\
j
\end{array}\right)}, \quad i=1, \ldots, n,
$$

where $j \in N$.

(e) OWA operators proposed by $\mathrm{Ahn}^{36}$ :

$$
\begin{aligned}
& w_{i}=\frac{1}{n} \sum_{j=i}^{n} \frac{1}{j}, \quad i=1, \ldots, n, \\
& w_{i}=\frac{2(n+1-i)}{n(n+1)}, \quad i=1, \ldots, n .
\end{aligned}
$$

(f) OWA operators based on exponential distribution ${ }^{37}$ :

$$
w_{i}=\frac{\mathrm{e}^{-i / \mu_{n}}}{\sum_{j=1}^{n} \mathrm{e}^{-j / \mu_{n}}}, \quad i=1, \ldots, n,
$$

where $\mu_{n}=\lambda(n+1)$ and $1 /(n+1) \leq \lambda \leq n /(n+1)$.

2. OWA operators associated with nondecreasing weighting vectors $\left(w_{1} \leq\right.$ $\left.w_{2} \leq \cdots \leq w_{n}\right)$. For instance:

(a) Andlike-window OWA operators ${ }^{28}$ :

$$
w_{i}= \begin{cases}0 & \text { if } i=1, \ldots, n-m \\ 1 / m & \text { otherwise }\end{cases}
$$


(b) Andlike S-OWA operators ${ }^{33}$ :

$$
w_{i}= \begin{cases}\frac{1-\lambda}{n} & \text { if } i=1, \ldots, n-1, \\ \lambda+\frac{1-\lambda}{n} & \text { otherwise }\end{cases}
$$

where $\lambda \in[0,1]$.

(c) Pessimistic exponential OWA operators ${ }^{34}$ :

$$
w_{i}= \begin{cases}\alpha^{n-1} & \text { if } i=1 \\ (1-\alpha) \alpha^{n-i} & \text { otherwise }\end{cases}
$$

where $\alpha \in[0,0.5]$.

(d) OWA operators proposed by $\mathrm{Ahn}^{36}$ :

$$
\begin{aligned}
& w_{i}=\frac{1}{n} \sum_{j=1}^{i} \frac{1}{n-j+1}, \quad i=1, \ldots, n, \\
& w_{i}=\frac{2 i}{n(n+1)}, \quad i=1, \ldots, n .
\end{aligned}
$$

(e) OWA operators based on the inverse form of exponential distribu$\operatorname{tion}^{37}$ :

$$
w_{i}=\frac{\mathrm{e}^{i / \mu_{n}}}{\sum_{j=1}^{n} \mathrm{e}^{j / \mu_{n}}}, \quad i=1, \ldots, n,
$$

where $\mu_{n}=\lambda(n+1)$ and $1 /(n+1) \leq \lambda \leq n /(n+1)$.

3. The binomial OWA operators introduced by León et al. ${ }^{38}$ :

$$
w_{i}=\left(\begin{array}{c}
n-1 \\
i-1
\end{array}\right)(1-\alpha)^{i-1} \alpha^{n-i}, \quad i=1, \ldots, n,
$$

where $\alpha \in[0,1] .^{\text {a }}$

4. OWA operators associated with weighting vectors obtained as a mixture of binomial and discrete uniform probabilities ${ }^{38}$ :

$$
w_{i}=\lambda\left(\begin{array}{c}
n-1 \\
i-1
\end{array}\right)(1-\alpha)^{i-1} \alpha^{n-i}+(1-\lambda) \frac{1}{n}, \quad i=1, \ldots, n,
$$

where $\alpha, \lambda \in[0,1]$.

5. OWA operators based on normal distribution ${ }^{37}$ :

$$
w_{i}=\frac{\mathrm{e}^{-\left(i-\mu_{n}\right)^{2} /\left(2 \sigma_{n}^{2}\right)}}{\sum_{j=1}^{n} \mathrm{e}^{-\left(j-\mu_{n}\right)^{2} /\left(2 \sigma_{n}^{2}\right)}}, \quad i=1, \ldots, n,
$$

where $\mu_{n}=\lambda(n+1), 1 /(n+1) \leq \lambda \leq n /(n+1)$, and $\left.\sigma_{n}^{2}=\frac{1}{n} \sum_{i=1}^{n}\left(i-\mu_{n}\right)^{2}\right)$.

${ }^{\text {a It }}$ is easy to check that these weights are strongly logarithmically concave and, consequently, unimodal. ${ }^{39}$ 
It is also worth noting that the optimal solutions of some methods proposed in the literature for choosing the weighting vector $\boldsymbol{w}$ have arithmetic or geometric form ${ }^{25,40}$; therefore they are nonincreasing or nondecreasing weighting vectors. Moreover, it is easy to check that the dual of a unimodal weighting vector is also unimodal. On the other hand, notice that when we add zeros at the beginning or end of a unimodal weighting vector we get another unimodal weighting vector (this is an easy procedure to obtain OWA operators located between two order statistics). So, this new family is very extensive and includes some of the most outstanding weighting vectors used in the literature of OWA operators.

The set of the weighting vectors corresponding to unimodal OWA operators will be denote by $\mathcal{W}_{\mathrm{u}}$.

\section{SUOWA OPERATORS CONSTRUCTED THROUGH UNIMODAL WEIGHTING VECTORS}

As noted in the introduction, the main weakness of SUOWA operators is that, sometimes, $v_{\boldsymbol{p}, \boldsymbol{w}}^{U}$ (the game associated with the weighting vectors $\boldsymbol{p}$ and $\boldsymbol{w}$ and the semiuninorm $U$ ) is not a capacity, and then it is necessary to calculate the monotonic cover of this game, $\hat{v}_{\boldsymbol{p}, \boldsymbol{w}}^{U}$. So, it is interesting to know the weighting vectors and semiuninorms that allow us to directly obtain a capacity. In this sense, some results on this issue have been given for several semiuninorms. ${ }^{10-12}$

In relation to the semiuninorm $U_{\min }^{\max }$, the game $v_{\boldsymbol{p}, \boldsymbol{w}}^{U_{\boldsymbol{m}}^{\max }}$ is given by

$$
\begin{aligned}
v_{\boldsymbol{p}, \boldsymbol{w}}^{U_{\min }^{\max }}(A)= & |A| U_{\min }^{\max }\left(\frac{\mu_{\boldsymbol{p}}(A)}{|A|}, \frac{\mu_{|\boldsymbol{w}|}(A)}{|A|}\right) \\
= & \begin{cases}\min \left(\sum_{i \in A} p_{i}, \sum_{i=1}^{|A|} w_{i}\right), & \text { if } \sum_{i=1}^{|A|} w_{i}<|A| / n, \\
\sum_{i \in A} p_{i}, & \text { if } \sum_{i=1}^{|A|} w_{i}=|A| / n, \\
\max \left(\sum_{i \in A} p_{i}, \sum_{i=1}^{|A|} w_{i}\right), & \text { if } \sum_{i=1}^{|A|} w_{i}>|A| / n,\end{cases}
\end{aligned}
$$

for any weighting vector $\boldsymbol{p}$ and $A \subseteq N$, with $|A| \geq 1$. In the analysis of the monotonicity of $v_{\boldsymbol{p}, \boldsymbol{w}}^{U_{\operatorname{win}}^{\max }}$, a first step was made by Llamazares, ${ }^{13}$ who proved that when $\boldsymbol{w}$ correspond to a window-OWA operator, the game $v_{\boldsymbol{p}, \boldsymbol{w}}^{U_{\operatorname{mix}}^{\max }}$ is a capacity.

Proposition 1. Let $\boldsymbol{w}$ be a weighting vector corresponding to a window-OWA operator. Then, for any weighting vector $\boldsymbol{p}, v_{\boldsymbol{p}, \boldsymbol{w}}^{U_{\operatorname{mix}}^{\max }}$ is a normalized capacity on $N$.

Now we generalize this result to unimodal weighting vectors. For this we will use the following lemma.

Lemma 1. Let $\boldsymbol{w} \in \mathcal{W}_{\mathrm{u}}$ and $q \in\{1, \ldots, n-1\}$.

1. If $q<n-1$ and $\sum_{i=1}^{q} w_{i}>q / n$, then $\sum_{i=1}^{q+1} w_{i}>(q+1) / n$. $^{\mathrm{b}}$

${ }^{\mathrm{b}}$ Notice that when $q=n-1, \sum_{i=1}^{q+1} w_{i}=1=(q+1) / n$. 
2. If $\sum_{i=1}^{q} w_{i}=q / n$, then $\sum_{i=1}^{q+1} w_{i} \geq(q+1) / n$.

Proof. We only proof the first statement, since the proof of the second one can be obtained in a similar way. Given $\boldsymbol{w} \in \mathcal{W}_{\mathrm{u}}$, there exists $k \in N$ such that $w_{1} \leq \cdots \leq w_{k-1} \leq w_{k} \geq w_{k+1} \geq \cdots \geq w_{n}$. We distinguish two cases:

1. If $q+1 \leq k$, then $w_{1} \leq \cdots \leq w_{q} \leq w_{q+1}$. Since $\sum_{i=1}^{q} w_{i}>q / n$ and these weights are nondecreasing, we have $w_{q}>1 / n$, and, consequently, $w_{q+1}>1 / n$. Therefore $\sum_{i=1}^{q+1} w_{i}>(q+1) / n$.

2. If $q+1>k$, then $w_{q+1} \geq w_{q+2} \geq \cdots \geq w_{n}$. Suppose, by contradiction, that $\sum_{i=1}^{q+1} w_{i} \leq(q+1) / n$. Then $\sum_{i=q+2}^{n} w_{i} \geq 1-(q+1) / n$. Moreover, since the weights $w_{q+1}, \ldots, w_{n}$ are nonincreasing, we have

$$
(n-(q+1)) w_{q+1} \geq \sum_{i=q+2}^{n} w_{i} \geq \frac{n-(q+1)}{n},
$$

that is, $w_{q+1} \geq 1 / n$. Since by hypothesis $\sum_{i=1}^{q} w_{i}>q / n$, we get $\sum_{i=1}^{q+1} w_{i}>$ $q / n+1 / n=(q+1) / n$, which contradicts the initial assumption.

Proposition 2. Given $\boldsymbol{w} \in \mathcal{W}_{\mathrm{u}}$, if we define

$$
\begin{array}{rlrl}
L_{\boldsymbol{w}} & =\left\{l \in N \mid \sum_{i=1}^{l} w_{i}<\frac{l}{n}\right\}, & L^{\boldsymbol{w}} & =\left\{l \in N \mid \sum_{i=1}^{l} w_{i}>\frac{l}{n}\right\}, \\
l_{\boldsymbol{w}} & = \begin{cases}0, & \text { if } L_{\boldsymbol{w}}=\varnothing, \\
\max L_{\boldsymbol{w}}, & \text { otherwise, }\end{cases} & l^{\boldsymbol{w}}= \begin{cases}n+1, & \text { if } L^{\boldsymbol{w}}=\varnothing, \\
\min L^{\boldsymbol{w}}, & \text { otherwise, }\end{cases}
\end{array}
$$

then $l_{\boldsymbol{w}}<l^{\boldsymbol{w}}$ and, for any weighting vector $\boldsymbol{p}, v_{\boldsymbol{p}, \boldsymbol{w}}^{U_{\operatorname{win}}^{\max }}$ is a normalized capacity on $N$ given by

$$
v_{\boldsymbol{p}, \boldsymbol{w}}^{U_{\min }^{\max }}(A)= \begin{cases}\min \left(\sum_{i \in A} p_{i}, \sum_{i=1}^{|A|} w_{i}\right), & \text { if }|A| \leq l_{\boldsymbol{w}}, \\ \sum_{i \in A} p_{i}, & \text { if } l_{\boldsymbol{w}}<|A|<l^{\boldsymbol{w}}, \\ \max \left(\sum_{i \in A} p_{i}, \sum_{i=1}^{|A|} w_{i}\right), & \text { if }|A| \geq l^{\boldsymbol{w}},\end{cases}
$$

where $A$ is any nonempty subset of $N$.

Proof. Let $\boldsymbol{w} \in \mathcal{W}_{\mathrm{u}}$. In order to show that $l_{\boldsymbol{w}}<l^{\boldsymbol{w}}$ we can suppose that $L^{\boldsymbol{w}} \neq \varnothing$ (if $L^{\boldsymbol{w}}=\varnothing$, then $l^{\boldsymbol{w}}=n+1>l_{\boldsymbol{w}}$ ). But, by the first item of Lemma 1 , $L^{\boldsymbol{w}}=\left\{l^{\boldsymbol{w}}, l^{\boldsymbol{w}}+1, \ldots, n-1\right\}$ and, since $n \notin L_{\boldsymbol{w}}$, we get $l_{\boldsymbol{w}}<l^{\boldsymbol{w}}$.

Notice also that if $L_{\boldsymbol{w}} \neq \varnothing$, then, by Lemma $1, L_{\boldsymbol{w}}=\left\{1, \ldots, l_{\boldsymbol{w}}\right\}$. So, given 
a weighting vector $\boldsymbol{p}$ and $\varnothing \subsetneq A \subseteq N$, by expression (4) we have

$$
\begin{aligned}
v_{\boldsymbol{p}, \boldsymbol{w}}^{U_{\min }^{\max }}(A) & = \begin{cases}\min \left(\sum_{i \in A} p_{i}, \sum_{i=1}^{|A|} w_{i}\right), & \text { if } \sum_{i=1}^{|A|} w_{i}<|A| / n, \\
\sum_{i \in A} p_{i}, & \text { if } \sum_{i=1}^{|A|} w_{i}=|A| / n, \\
\max \left(\sum_{i \in A} p_{i}, \sum_{i=1}^{|A|} w_{i}\right), & \text { if } \sum_{i=1}^{|A|} w_{i}>|A| / n,\end{cases} \\
= & \begin{cases}\min \left(\sum_{i \in A} p_{i}, \sum_{i=1}^{|A|} w_{i}\right), & \text { if }|A| \leq l_{\boldsymbol{w}}, \\
\sum_{i \in A} p_{i}, & \text { if } l_{\boldsymbol{w}}<|A|<l^{\boldsymbol{w}}, \\
\max \left(\sum_{i \in A} p_{i}, \sum_{i=1}^{|A|} w_{i}\right), & \text { if }|A| \geq l^{\boldsymbol{w}} .\end{cases}
\end{aligned}
$$

Lastly, in order to prove the monotonicity of $v_{\boldsymbol{p}, \boldsymbol{w}}^{U_{\operatorname{mix}}^{\max }}$ it is sufficient to show that $v_{\boldsymbol{p}, \boldsymbol{w}}^{U_{\operatorname{mix}}^{\max }}(A) \leq v_{\boldsymbol{p} \text {, } \boldsymbol{w}}^{U_{\min }^{\max }}(B)$ for any $A \subsetneq N$ and $B=A \cup\{j\}$, with $j \notin A$. Since the case $A=\varnothing$ is trivial, we suppose that $|A| \geq 1$. We distinguish three cases:

1. If $|A| \leq l_{\boldsymbol{w}}$, then

$$
v_{\boldsymbol{p}, \boldsymbol{w}}^{U_{\min }^{\max }}(A)=\min \left(\sum_{i \in A} p_{i}, \sum_{i=1}^{|A|} w_{i}\right) \leq \min \left(\sum_{i \in B} p_{i}, \sum_{i=1}^{|B|} w_{i}\right) \leq v_{\boldsymbol{p}, \boldsymbol{w}}^{U_{\min }^{\max }}(B) .
$$

2. If $l_{\boldsymbol{w}}<|A|<l^{\boldsymbol{w}}$, then

$$
v_{\boldsymbol{p}, \boldsymbol{w}}^{U_{\min }^{\max }}(A)=\sum_{i \in A} p_{i} \leq \sum_{i \in B} p_{i} \leq v_{\boldsymbol{p}, \boldsymbol{w}}^{U_{\min }^{\max }}(B)
$$

3. If $|A| \geq l^{w}$, then

$$
v_{\boldsymbol{p}, \boldsymbol{w}}^{U_{\operatorname{win}}^{\max }}(A)=\max \left(\sum_{i \in A} p_{i}, \sum_{i=1}^{|A|} w_{i}\right) \leq \max \left(\sum_{i \in B} p_{i}, \sum_{i=1}^{|B|} w_{i}\right)=v_{\boldsymbol{p}, \boldsymbol{w}}^{U_{\operatorname{mix}}^{\max }}(B) .
$$

So, given $\boldsymbol{w} \in \mathcal{W}_{\mathrm{u}}$, the values $l_{\boldsymbol{w}}$ and $l^{\boldsymbol{w}}$, together with expression (5), allow us to know explicitly the capacity $v_{\boldsymbol{p}, \boldsymbol{w}}^{U_{\min }^{\max }}$. Next we are going to give the values of $l_{\boldsymbol{w}}$ and $l^{\boldsymbol{w}}$ for the unimodal weighting vectors showed in Section 4 .

1. Nonincreasing weighting vectors where $w_{1}>1 / n$ (notice that if $w_{1}=1 / n$ then $\boldsymbol{w}=\boldsymbol{\eta}$, and, therefore, $\left.v_{\boldsymbol{p}, \boldsymbol{w}}^{U_{\operatorname{mix}}^{\max }}=\mu_{\boldsymbol{p}}\right)$ : It is easy to check that $l_{\boldsymbol{w}}=0$ and $l^{\boldsymbol{w}}=1$. So, for any weighting vector $\boldsymbol{p}$ and $A \subseteq N$, with $|A| \geq 1$, we have

$$
v_{\boldsymbol{p}, \boldsymbol{w}}^{U_{\min }^{\max }}(A)=\max \left(\sum_{i \in A} p_{i}, \sum_{i=1}^{|A|} w_{i}\right)
$$


Moreover, notice that if for any $j \in\{1, \ldots, n-1\}, \sum_{i=1}^{j} w_{i} \geq \sum_{U^{\max }}^{\sum_{i=1}^{j}} p_{[i]}$ (where $p_{[1]} \geq p_{[2]} \geq \cdots \geq p_{[n]}$ ), then $v_{\boldsymbol{p}, \boldsymbol{w}}^{U_{\min }^{\max }}=\mu_{|\boldsymbol{w}|}$, that is, $S_{\boldsymbol{p}, \boldsymbol{w}}^{U_{\operatorname{mix}}^{\max }}=O_{\boldsymbol{w}}$. For instance, consider the Orlike-window OWA operator given by

$$
w_{i}= \begin{cases}1 / m & \text { if } i=1, \ldots, m \\ 0 & \text { otherwise }\end{cases}
$$

with $m<n$, and let $\boldsymbol{p}$ be a weighting vector such that $\max _{i=1, \ldots, n} p_{i} \leq 1 / m$. Then, the SUOWA operator associated with $\boldsymbol{p}, \boldsymbol{w}$ and $U_{\min }^{\max }$ coincides with the OWA operator associated with $\boldsymbol{w}$.

2. Nondecreasing weighting vectors where $w_{1}<1 / n$ (notice that if $w_{1}=1 / n$ then $\boldsymbol{w}=\boldsymbol{\eta}$, and, therefore, $\left.v_{\boldsymbol{p}, \boldsymbol{w}}^{U_{\min }^{\max }}=\mu_{\boldsymbol{p}}\right)$ : It is easy to check that $l_{\boldsymbol{w}}=n-1$ and $l^{\boldsymbol{w}}=n+1$. So, for any weighting vector $\boldsymbol{p}$ and $A \subseteq N,|A| \geq 1$, we have

$$
v_{\boldsymbol{p}, \boldsymbol{w}}^{U_{\operatorname{mix}}^{\max }}(A)=\min \left(\sum_{i \in A} p_{i}, \sum_{i=1}^{|A|} w_{i}\right) \text {. }
$$

Moreover, notice that if for any $j \in\{1, \ldots, n-1\}, \sum_{i=1}^{j} w_{i} \leq \sum_{i=1}^{j} p_{(i)}$ (where $\left.p_{(1)} \leq p_{(2)} \leq \cdots \leq p_{(n)}\right)$, then $v_{\boldsymbol{p}, \boldsymbol{w}}^{U_{\min }^{\max }}=\mu_{|\boldsymbol{w}|}$, that is, $S_{\boldsymbol{p}, \boldsymbol{w}}^{U_{\min }^{\max }}=O_{\boldsymbol{w}}$. For instance, consider the Andlike-window OWA operator given by

$$
w_{i}= \begin{cases}0 & \text { if } i=1, \ldots, n-m \\ 1 / m & \text { otherwise }\end{cases}
$$

with $m<n$, and let $\boldsymbol{p}$ be a weighting vector such that $\max _{i=1, \ldots, n} p_{i} \leq 1 / m$. Then, the SUOWA operator associated with $\boldsymbol{p}, \boldsymbol{w}$ and $U_{\min }^{\max }$ coincides with the OWA operator associated with $\boldsymbol{w}$.

3. Weighting vectors associated with window-OWA operators such that $\boldsymbol{w}$ is neither nonincreasing nor nondecreasing; that is,

$$
w_{i}= \begin{cases}1 / m & \text { if } i=r, \ldots, r+m-1 \\ 0 & \text { otherwise }\end{cases}
$$

where $r, m \in N$, with $r \geq 2$ and $r+m \leq n$. Notice that, given $l \geq r$,

$\sum_{i=1}^{l} w_{i}<\frac{l}{n} \Leftrightarrow \frac{1}{m}(l-r+1)<\frac{l}{n} \Leftrightarrow l(n-m)<n(r-1) \Leftrightarrow l<\frac{n(r-1)}{n-m}$,

and, consequently,

$$
l_{\boldsymbol{w}}=\left\lceil\frac{n(r-1)}{n-m}\right\rceil-1=\left\lceil\frac{n(r-2)+m}{n-m}\right\rceil .
$$

Analogously,

$$
\sum_{i=1}^{l} w_{i}>\frac{l}{n} \Leftrightarrow l>\frac{n(r-1)}{n-m}
$$


and, then

$$
l^{\boldsymbol{w}}=\left\lfloor\frac{n(r-1)}{n-m}\right\rfloor+1=\left\lfloor\frac{n r-m}{n-m}\right\rfloor .
$$

4. Weighting vectors associated with centered OWA operators: It is straightforward to check that the values taken by $l_{\boldsymbol{w}}$ and $l^{\boldsymbol{w}}$ are the following:

(a) If $n$ is odd: $l_{\boldsymbol{w}}=(n-1) / 2$ and $l^{\boldsymbol{w}}=(n+1) / 2$.

(b) If $n$ is even: $l_{\boldsymbol{w}}=n / 2-1$ and $l^{\boldsymbol{w}}=n / 2+1$.

Next we are going to show some interesting families of functions obtained as SUOWA operators associated with unimodal weighting vectors. Consider the weighting vector $\boldsymbol{w}$ defined by

$$
w_{i}= \begin{cases}0 & \text { if } i=1, \ldots, r-1 \\ \frac{r}{n} & \text { if } i=r \\ \frac{1}{n} & \text { otherwise }\end{cases}
$$

where $r \in N$. Note that if $r=1$ then $O_{\boldsymbol{w}}$ is the arithmetic mean, and if $r=n$ then $O_{\boldsymbol{w}}$ is the minimum. When $r \in\{2, \ldots, n-1\}$, the behavior of $O_{\boldsymbol{w}}$ is similar to that of the winsorized means (see, for instance, Beliakov et al. ${ }^{3}$, p. 103), but only replacing the $r-1$ highest values by $x_{[r]}$. It is straightforward to check that $l_{\boldsymbol{w}}=r-1$ and $l^{\boldsymbol{w}}=n+1$, and, consequently,

$$
v_{\boldsymbol{p}, \boldsymbol{w}}^{U_{\operatorname{win}}^{\max }}(A)= \begin{cases}0, & \text { if }|A|<r \\ \sum_{i \in A} p_{i}, & \text { if }|A| \geq r\end{cases}
$$

for any weighting vector $\boldsymbol{p}$ and $A \subseteq N$, with $|A| \geq 1$. Therefore, the SUOWA operator associated with $\boldsymbol{p}, \boldsymbol{w}$ and $U_{\min }^{\max }$ is defined by

$$
S_{\boldsymbol{p}, \boldsymbol{w}}^{U_{\min }^{\max }}(\boldsymbol{x})=\sum_{i=1}^{n}\left(v_{\boldsymbol{p}, \boldsymbol{w}}^{U_{\min }^{\max }}\left(A_{[i]}\right)-v_{\boldsymbol{p}, \boldsymbol{w}}^{U_{\min }^{\max }}\left(A_{[i-1]}\right)\right) x_{[i]}=\left(\sum_{i=1}^{r} p_{[i]}\right) x_{[r]}+\sum_{i=r+1}^{n} p_{[i]} x_{[i]},
$$

that is, it is the weighted mean associated with $\boldsymbol{p}$ where the $r-1$ highest values have been previously replaced by $x_{[r]}$. Notice that these functions seem the natural extension of $O_{\boldsymbol{w}}$ when the weighting vector $\boldsymbol{p}$ is introduced in the aggregation process. Likewise, note that in the extreme cases $r=1$ and $r=n$, the operator $S_{\boldsymbol{p}, \boldsymbol{w}}^{U_{\min }^{\max }}$ coincides with the weighted mean associated with $\boldsymbol{p}$ and the minimum, respectively.

Analogously, when we consider the weighting vector $\boldsymbol{w}$ defined by

$$
w_{i}= \begin{cases}\frac{1}{n} & \text { if } i=1, \ldots, n-r^{\prime} \\ \frac{r^{\prime}}{n} & \text { if } i=n-r^{\prime}+1 \\ 0 & \text { otherwise }\end{cases}
$$


where $r^{\prime} \in N$, the OWA operator associated with $\boldsymbol{w}$ is an arithmetic mean where the $r^{\prime}-1$ lowest values have been previously replaced by $x_{\left[n-r^{\prime}+1\right]}=x_{\left(r^{\prime}\right)}$. In this case, $l_{\boldsymbol{w}}=0$ and $l^{\boldsymbol{w}}=n-r^{\prime}+1$, and

$$
v_{\boldsymbol{p}, \boldsymbol{w}}^{U_{\min }^{\max }}(A)= \begin{cases}\sum_{i \in A} p_{i}, & \text { if }|A| \leq n-r^{\prime} \\ 1, & \text { if }|A|>n-r^{\prime}\end{cases}
$$

for any weighting vector $\boldsymbol{p}$ and $A \subseteq N$, with $|A| \geq 1$. Hence,

$$
\begin{aligned}
S_{\boldsymbol{p}, \boldsymbol{w}}^{U_{\min }^{\max }}(\boldsymbol{x}) & =\sum_{i=1}^{n}\left(v_{\boldsymbol{p}, \boldsymbol{w}}^{U_{\operatorname{mix}}^{\max }}\left(A_{[i]}\right)-v_{\boldsymbol{p}, \boldsymbol{w}}^{U_{\operatorname{mix}}^{\max }}\left(A_{[i-1]}\right)\right) x_{[i]} \\
& =\sum_{i=1}^{n-r^{\prime}} p_{[i]} x_{[i]}+\left(\sum_{i=n-r^{\prime}+1}^{n} p_{[i]}\right) x_{\left[n-r^{\prime}+1\right]},
\end{aligned}
$$

that is, it is the weighted mean associated with $\boldsymbol{p}$ where the $r^{\prime}-1$ lowest values have been previously replaced by $x_{\left[n-r^{\prime}+1\right]}=x_{\left(r^{\prime}\right)}$.

Finally, consider the weighting vector $\boldsymbol{w}$ defined by

$$
w_{i}= \begin{cases}0 & \text { if } i=1, \ldots, r-1, \\ \frac{r}{n} & \text { if } i=r, \\ \frac{1}{n} & \text { if } i=r+1, \ldots, n-r^{\prime} \\ \frac{r^{\prime}}{n} & \text { if } i=n-r^{\prime}+1, \\ 0 & \text { otherwise, }\end{cases}
$$

where $r, r^{\prime} \in N$, with $r+r^{\prime} \leq n$. Notice that when $r=r^{\prime}>1$, the OWA operator associated with $\boldsymbol{w}$ is a winsorized mean. Likewise, note that in this case $\boldsymbol{w}$ is not a unimodal weighting vector. However, from expression (4), it is easy to check that $v_{\boldsymbol{p}, \boldsymbol{w}}^{U_{\operatorname{mix}}^{\max }}$ is a normalized capacity given by

$$
v_{\boldsymbol{p}, \boldsymbol{w}}^{U_{\min }^{\max }}(A)= \begin{cases}0, & \text { if }|A|<r, \\ \sum_{i \in A} p_{i}, & \text { if } r \leq|A| \leq n-r^{\prime}, \\ 1, & \text { if }|A|>n-r^{\prime},\end{cases}
$$

for any weighting vector $\boldsymbol{p}$ and $A \subseteq N$, with $|A| \geq 1$. So,

$$
\begin{aligned}
S_{\boldsymbol{p}, \boldsymbol{w}}^{U_{\min }^{\max }}(\boldsymbol{x}) & =\sum_{i=1}^{n}\left(v_{\boldsymbol{p}, \boldsymbol{w}}^{U_{\min }^{\max }}\left(A_{[i]}\right)-v_{\boldsymbol{p}, \boldsymbol{w}}^{U_{\min }^{\max }}\left(A_{[i-1]}\right)\right) x_{[i]} \\
& =\left(\sum_{i=1}^{r} p_{[i]}\right) x_{[r]}+\sum_{i=r+1}^{n-r^{\prime}} p_{[i]} x_{[i]}+\left(\sum_{i=n-r^{\prime}+1}^{n} p_{[i]}\right) x_{\left[n-r^{\prime}+1\right]},
\end{aligned}
$$

that is, it is the weighted mean associated with $\boldsymbol{p}$ where the $r-1$ highest values and the $r^{\prime}-1$ lowest values have been previously replaced by $x_{[r]}$ and $x_{\left[n-r^{\prime}+1\right]}=$ $x_{\left(r^{\prime}\right)}$, respectively. 


\section{ANALYSIS OF SOME PROPERTIES}

In this section we show some properties that the SUOWA operators constructed by using $U_{\min }^{\max }$ and unimodal weighting vectors satisfy. Firstly we list some properties that are already known for idempotent semiuninorms, rewritten for $U_{\min }^{\max }$ and unimodal weighting vectors. ${ }^{7,10}$ It is worth noting that, given that SUOWA operators appears in the literature with the aim of combining weighted means and OWA operators in a single function, it seems appropriate to establish a relation between the capacities of SUOWA operators and those of weighted means and OWA operators. In this regard, it should be noted that, for any subset $A$ of $N$, the value $v_{\boldsymbol{p}, \boldsymbol{w}}^{U_{\min }^{\max }}(A)$ range between the values $\mu_{\boldsymbol{p}}(A)$ and $\mu_{|\boldsymbol{w}|}(A)$.

Proposition 3. Let $\boldsymbol{w} \in \mathcal{W}_{\mathrm{u}}$ and let $\boldsymbol{p}$ be a weighting vector. Then:

1. Given $A \subseteq N$, $\min \left(\mu_{\boldsymbol{p}}(A), \mu_{|\boldsymbol{w}|}(A)\right) \leq v_{\boldsymbol{p}, \boldsymbol{w}}^{U_{\min }^{\max }}(A) \leq \max \left(\mu_{\boldsymbol{p}}(A), \mu_{|\boldsymbol{w}|}(A)\right)$.

2. If $\mu_{\boldsymbol{p}} \leq \mu_{|\boldsymbol{w}|}$, then $M_{\boldsymbol{p}} \leq S_{\boldsymbol{p}, \boldsymbol{w}}^{U_{\min }^{\max }} \leq O_{\boldsymbol{w}}$; if $\mu_{|\boldsymbol{w}|} \leq \mu_{\boldsymbol{p}}$, then $O_{\boldsymbol{w}} \leq S_{\boldsymbol{p}, \boldsymbol{w}}^{U_{\min }^{\max }} \leq$ $M_{p}$.

A similar result to that shown in the first item of the previous proposition can be established for the weight $s_{i}=v_{\boldsymbol{p}, \boldsymbol{w}}^{U_{\operatorname{mix}}^{\max }}\left(A_{[i]}\right)-v_{\boldsymbol{p}, \boldsymbol{w}}^{U_{\min }^{\max }}\left(A_{[i-1]}\right)$, which affecting the component $x_{[i]}$ of $\boldsymbol{x}$ : when we consider the semiuninorm $U_{\min }^{\max }$ and unimodal weighting vectors, the weight $s_{i}$ is located between $p_{[i]}$ and $w_{i}$.

Proposition 4. Let $\boldsymbol{w} \in \mathcal{W}_{\mathrm{u}}$ and let $\boldsymbol{p}$ be a weighting vector. Then, for any $\boldsymbol{x} \in \mathbb{R}^{n}$ and $i \in N$ we have $\min \left(p_{[i]}, w_{i}\right) \leq s_{i} \leq \max \left(p_{[i]}, w_{i}\right)$.

Proof. By Proposition 2, given $\boldsymbol{x} \in \mathbb{R}^{n}$ and $i \in N$, the weight $s_{i}$ could take one of the following values:

1. $\min \left(\mu_{\boldsymbol{p}}\left(A_{[i]}\right), \mu_{|\boldsymbol{w}|}\left(A_{[i]}\right)\right)-\min \left(\mu_{\boldsymbol{p}}\left(A_{[i-1]}\right), \mu_{|\boldsymbol{w}|}\left(A_{[i-1]}\right)\right)$.

2. $\mu_{\boldsymbol{p}}\left(A_{[i]}\right)-\min \left(\mu_{\boldsymbol{p}}\left(A_{[i-1]}\right), \mu_{|\boldsymbol{w}|}\left(A_{[i-1]}\right)\right)$.

3. $\max \left(\mu_{\boldsymbol{p}}\left(A_{[i]}\right), \mu_{|\boldsymbol{w}|}\left(A_{[i]}\right)\right)-\min \left(\mu_{\boldsymbol{p}}\left(A_{[i-1]}\right), \mu_{|\boldsymbol{w}|}\left(A_{[i-1]}\right)\right)$.

4. $\mu_{\boldsymbol{p}}\left(A_{[i]}\right)-\mu_{\boldsymbol{p}}\left(A_{[i-1]}\right)$.

5. $\max \left(\mu_{\boldsymbol{p}}\left(A_{[i]}\right), \mu_{|\boldsymbol{w}|}\left(A_{[i]}\right)\right)-\mu_{\boldsymbol{p}}\left(A_{[i-1]}\right)$.

6. $\max \left(\mu_{\boldsymbol{p}}\left(A_{[i]}\right), \mu_{|\boldsymbol{w}|}\left(A_{[i]}\right)\right)-\max \left(\mu_{\boldsymbol{p}}\left(A_{[i-1]}\right), \mu_{|\boldsymbol{w}|}\left(A_{[i-1]}\right)\right)$.

The study of the previous cases comes down to the analysis of the following four values that may take $s_{i}$ :

1. $\mu_{\boldsymbol{p}}\left(A_{[i]}\right)-\mu_{\boldsymbol{p}}\left(A_{[i-1]}\right)=p_{[i]}$,

2. $\mu_{\boldsymbol{p}}\left(A_{[i]}\right)-\mu_{|\boldsymbol{w}|}\left(A_{[i-1]}\right)$,

3. $\mu_{|\boldsymbol{w}|}\left(A_{[i]}\right)-\mu_{\boldsymbol{p}}\left(A_{[i-1]}\right)$, 
4. $\mu_{|\boldsymbol{w}|}\left(A_{[i]}\right)-\mu_{|\boldsymbol{w}|}\left(A_{[i-1]}\right)=w_{i}$,

which has been carried out in Llamazares ${ }^{10}$, p. 843 for the semiuninorm $U_{\text {min }}$. In any of the four cases, $\min \left(p_{[i]}, w_{i}\right) \leq s_{i} \leq \max \left(p_{[i]}, w_{i}\right)$.

We now consider the properties of balancing, subadditivity and superadditivity. Balancing property was introduced by Calvo et al. ${ }^{41}$, and it guarantees the monotonicity with respect the cardinality of the sets. On the other hand, subadditivity and superadditivity properties are well known in the literature of games and capacities.

DeFinition 13. Let $\mu$ be a normalized capacity.

1. $\mu$ is balanced if $|A|<|B| \Rightarrow \mu(A) \leq \mu(B)$ for all $A, B \subseteq N$.

2. $\mu$ is subadditive if $\mu(A \cup B) \leq \mu(A)+\mu(B)$ for all $A, B \subseteq N$ such that $A \cap B=\varnothing$.

3. $\mu$ is superadditive if $\mu(A \cup B) \geq \mu(A)+\mu(B)$ for all $A, B \subseteq N$ such that $A \cap B=\varnothing$.

It is easy to check that $v_{\boldsymbol{p} \text {, w }}^{U_{\operatorname{mix}}^{\max }}$ is balanced. As we show below, the property of subadditivity (superadditivity) is satisfied when the weighting vector $\boldsymbol{w}$ is nonincreasing (nondecreasing).

Proposition 5. Let $\boldsymbol{w} \in \mathcal{W}_{\mathrm{u}}$ and let $\boldsymbol{p}$ be a weighting vector.

1. If $\boldsymbol{w}$ is a nonincreasing weighting vector, then $v_{\boldsymbol{p}, \boldsymbol{w}}^{U_{\min }^{\max }}$ is subadditive.

2. If $\boldsymbol{w}$ is a nondecreasing weighting vector, then $v_{\boldsymbol{p}, \boldsymbol{w}}^{U_{\min }^{\max }}$ is superadditive.

Proof. We will only prove the first statement, because the other one can be obtained in a similar way. Let $\boldsymbol{w}$ be a nonincreasing weighting vector and let $\boldsymbol{p}$ be a weighting vector. Given $A, B \subseteq N$ such that $A \cap B=\varnothing$, note that, since $\boldsymbol{w}$ is nonincreasing,

$$
\sum_{i=1}^{|A|+|B|} w_{i} \leq \sum_{i=1}^{|A|} w_{i}+\sum_{i=1}^{|B|} w_{i}
$$

Therefore,

$$
\begin{aligned}
v_{\boldsymbol{p}, \boldsymbol{w}}^{U_{\operatorname{mix}}^{\max }}(A \cup B) & =\max \left(\sum_{i \in A \cup B} p_{i}, \sum_{i=1}^{|A|+|B|} w_{i}\right) \\
& \leq \max \left(\sum_{i \in A} p_{i}, \sum_{i=1}^{|A|} w_{i}\right)+\max \left(\sum_{i \in B} p_{i}, \sum_{i=1}^{|B|} w_{i}\right) \\
& =v_{\boldsymbol{p}, \boldsymbol{w}}^{U_{\min }^{\max }}(A)+v_{\boldsymbol{p}, \boldsymbol{w}}^{U_{\operatorname{wix}}^{\max }}(B) .
\end{aligned}
$$


The last property studied refers to the duality: As in OWA operators, the dual capacity of $v_{\boldsymbol{p}, \boldsymbol{w}}^{U_{\min }^{\max }}$ can be easily obtained by using the dual of the weighting vector $\boldsymbol{w}$.

PROPOSITION 6. Let $\boldsymbol{w} \in \mathcal{W}_{\mathrm{u}}$. Then, for any weighting vector $\boldsymbol{p}, \bar{v}_{\boldsymbol{p}, \boldsymbol{w}}^{U_{\operatorname{mix}}^{\max }}=v_{\boldsymbol{p}, \boldsymbol{w}}^{U_{\min }^{\max }}$.

Proof. Let $\boldsymbol{w} \in \mathcal{W}_{\mathrm{u}}$ and let $\boldsymbol{p}$ a weighting vector. Given $\varnothing \subsetneq A \subseteq N$ (the case $A=\varnothing$ is trivial), notice that $\left|A^{\mathrm{c}}\right|=n-|A|$ and $\sum_{i=1}^{\left|A^{\mathrm{c}}\right|} w_{i}+\sum_{i=1}^{|\bar{A}|} \bar{w}_{i}=1$. Therefore

$$
\sum_{i=1}^{\left|A^{\mathrm{c}}\right|} w_{i}<\frac{\left|A^{\mathrm{c}}\right|}{n} \Leftrightarrow 1-\sum_{i=1}^{|A|} \bar{w}_{i}<1-\frac{|A|}{n} \Leftrightarrow \sum_{i=1}^{|A|} \bar{w}_{i}>\frac{|A|}{n} .
$$

Analogously,

$$
\begin{aligned}
& \sum_{i=1}^{\left|A^{\mathrm{c}}\right|} w_{i}=\frac{\left|A^{\mathrm{c}}\right|}{n} \Leftrightarrow \sum_{i=1}^{|A|} \bar{w}_{i}=\frac{|A|}{n}, \\
& \sum_{i=1}^{\left|A^{\mathrm{c}}\right|} w_{i}>\frac{\left|A^{\mathrm{c}}\right|}{n} \Leftrightarrow \sum_{i=1}^{|A|} \bar{w}_{i}<\frac{|A|}{n} .
\end{aligned}
$$

Now, by Proposition 2, $v_{\boldsymbol{p}, \boldsymbol{w}}^{U_{\min }^{\max }}$ is a normalized capacity on $N$ given by expression (4). Since, given $A \subseteq N, \bar{v}_{\boldsymbol{p}, \boldsymbol{w}}^{U_{\text {max }}^{\max }}(A)=1-v_{\boldsymbol{p}, \boldsymbol{w}}^{U_{\min }^{\max }}\left(A^{\mathrm{c}}\right)$, we distinguish three cases:

1. If $\sum_{i=1}^{\left|A^{\mathrm{c}}\right|} w_{i}<\left|A^{\mathrm{c}}\right| / n$, then

$$
\bar{v}_{\boldsymbol{p}, \boldsymbol{w}}^{U_{\min }^{\max }}(A)=1-\min \left(\sum_{i \in A^{c}} p_{i}, \sum_{i=1}^{\left|A^{c}\right|} w_{i}\right)=\max \left(\sum_{i \in A} p_{i}, \sum_{i=1}^{|A|} \bar{w}_{i}\right)=v_{\boldsymbol{p}, \overline{\boldsymbol{w}}}^{U_{\min }^{\max }}(A) .
$$

2. If $\sum_{i=1}^{\left|A^{\mathrm{c}}\right|} w_{i}=\left|A^{\mathrm{c}}\right| / n$, then

$$
\bar{v}_{\boldsymbol{p}, \boldsymbol{w}}^{U_{\min }^{\max }}(A)=1-\sum_{i \in A^{\mathrm{c}}} p_{i}=\sum_{i \in A} p_{i}=v_{\boldsymbol{p}, \overline{\boldsymbol{w}}}^{U_{\min }^{\max }}(A) .
$$

3. If $\sum_{i=1}^{\left|A^{\mathrm{c}}\right|} w_{i}>\left|A^{\mathrm{c}}\right| / n$, then

$$
\bar{v}_{\boldsymbol{p}, \boldsymbol{w}}^{U_{\operatorname{mix}}^{\max }}(A)=1-\max \left(\sum_{i \in A^{c}} p_{i}, \sum_{i=1}^{\left|A^{c}\right|} w_{i}\right)=\min \left(\sum_{i \in A} p_{i}, \sum_{i=1}^{|A|} \bar{w}_{i}\right)=v_{\boldsymbol{p}, \overline{\boldsymbol{w}}}^{U_{\min }^{\max }}(A) .
$$

We finally emphasize that the SUOWA operators constructed by using the semiuninorm $U_{\min }^{\max }$ preserve the conjunctive/disjuntive character of the OWA operator associated with them. ${ }^{13}$ In this way, it is possible to get operators located between two order statistics that take into account the weights of the criteria.

Proposition 7. Let $\boldsymbol{w}$ be a weighting vector. If there exist $k, k^{\prime} \in N$ such that $\mathrm{OS}_{k} \leq O_{\boldsymbol{w}} \leq \mathrm{OS}_{k^{\prime}}$, then $\mathrm{OS}_{k} \leq S_{\boldsymbol{p} \boldsymbol{w} \boldsymbol{w}}^{U_{\min }} \leq \mathrm{OS}_{k^{\prime}}$ for any weighting vector $\boldsymbol{p}$. 


\section{CONCLUDING REMARKS}

SUOWA operators were introduced in the literature for generalizing simultaneously weighted means and OWA operators. In this way it is possible to deal with problems where both the significance of the information sources and the importance of the relative size of the values have to be taken into account. Nevertheless, the main weakness of SUOWA operators is that, sometimes, the game used in their construction is not monotonic and it is necessary to calculate its monotonic cover.

In this paper we have shown that when using unimodal weighting vectors and the semiuninorm $U_{\min }^{\max }$ we directly get normalized capacities. Given that unimodal weighting vectors embrace a great variety of weighting vectors, we can avoid the calculation of the monotonic cover on numerous occasions. We next summarize some important features of these capacities which are very appealing in practice:

1. Their expression is very simple, which greatly facilitates their use.

2. Their dual capacities are obtained by means of the dual of the weighting vectors $\boldsymbol{w}$.

3. The SUOWA operators associated with them satisfy that the weights $s_{i}$, which affect the values $x_{[i]}$, range between the weights $p_{[i]}$ and $w_{i}$.

4. The SUOWA operators associated with them maintain the conjuctive/disjunctive character of the OWA operators. In this way, it is possible to obtain functions located between two order statistics that take into account the weights of the information sources (see, for instance, the last part of Section 5).

\section{Acknowledgments}

This work is partially supported by the Spanish Ministry of Economy and Competitiveness (Project ECO2016-77900-P).

\section{References}

1. Beliakov G, Pradera A, Calvo T. Aggregation Functions: A Guide for Practitioners, Studies in Fuzziness and Soft Computing, vol. 221. Berlin: Springer, 2007.

2. Grabisch M, Marichal J, Mesiar R, Pap E. Aggregation Functions. Cambridge: Cambridge University Press, 2009.

3. Beliakov G, Bustince H, Calvo T. A Practical Guide to Averaging Functions, Studies in Fuzziness and Soft Computing, vol. 329. Cham: Springer, 2016. 
4. Yager RR. On ordered weighted averaging operators in multicriteria decision making. IEEE Trans Syst, Man, Cybern 1988;18:183-190.

5. Llamazares B. An analysis of some functions that generalizes weighted means and OWA operators. Int J Intell Syst 2013;28:380-393.

6. Torra V. The weighted OWA operator. Int J Intell Syst 1997;12:153-166.

7. Llamazares B. Constructing Choquet integral-based operators that generalize weighted means and OWA operators. Inform Fusion 2015;23:131-138.

8. Beliakov G. A method of introducing weights into OWA operators and other symmetric functions. In: Kreinovich V, editor, Uncertainty Modeling: Dedicated to Professor Boris Kovalerchuk on his Anniversary, 37-52. Cham: Springer, 2017.

9. Llamazares B. A study of SUOWA operators in two dimensions. Math Probl Eng 2015;2015:Article ID 271491, 12 pages.

10. Llamazares B. A behavioral analysis of WOWA and SUOWA operators. Int J Intell Syst 2016;31:827-851.

11. Llamazares B. SUOWA operators: Constructing semi-uninorms and analyzing specific cases. Fuzzy Sets Syst 2016;287:119-136.

12. Llamazares B. Closed-form expressions for some indices of SUOWA operators. Inform Fusion 2018;41:80-90.

13. Llamazares B. SUOWA operators: An analysis of their conjunctive/disjunctive character. Submitted 2017.

14. Liu HW. Semi-uninorms and implications on a complete lattice. Fuzzy Sets Syst 2012;191:72-82.

15. Choquet G. Theory of capacities. Ann Inst Fourier 1953;5:131-295.

16. Sugeno M. Theory of Fuzzy Integrals and its Applications. PhD thesis, Tokyo Institute of Technology, 1974.

17. Maschler M, Peleg B. The structure of the kernel of a cooperative game. SIAM J Appl Math 1967;15:569-604.

18. Maschler M, Peleg B, Shapley LS. The kernel and bargaining set for convex games. Int J Game Theory 1971;1:73-93.

19. Denneberg D. Non-Additive Measures and Integral. Dordrecht: Kluwer Academic Publisher, 1994. 
20. Torra V. On some relationships between the WOWA operator and the Choquet integral. In: Proc. 7th Int. Conf. on Information Processing and Management of Uncertainty in Knowledge-Based Systems (IPMU'98); 1998. pp 818-824.

21. Fodor J, Marichal JL, Roubens M. Characterization of the ordered weighted averaging operators. IEEE Trans Fuzzy Syst 1995;3:236-240.

22. Grabisch M. Fuzzy integral in multicriteria decision making. Fuzzy Sets Syst 1995;69:279-298.

23. Grabisch M. On equivalence classes of fuzzy connectives - the case of fuzzy integrals. IEEE Trans Fuzzy Syst 1995;3:96-109.

24. Yager RR, Rybalov A. Uninorm aggregation operators. Fuzzy Sets Syst 1996;80:111-120.

25. Liu X. A review of the OWA determination methods: Classification and some extensions. In: Yager RR, Kacprzyk J, Beliakov G, editors, Recent Developments in the Ordered Weighted Averaging Operators: Theory and Practice, 49-90. Berlin: Springer, 2011.

26. Wang J, Merigó JM, Jin L. S-H OWA operators with moment measure. Int J Intell Syst 2017;32:51-66.

27. Bai C, Zhang R, Song C, Wu Y. A new ordered weighted averaging operator to obtain the associated weights based on the principle of least mean square errors. Int J Intell Syst 2017;32:213-226.

28. Yager RR. Families of OWA operators. Fuzzy Sets Syst 1993;59:125-148.

29. Yager RR. Centered OWA operators. Soft Comput 2007;11:631-639.

30. Yager RR. Quantifier guided aggregation using OWA operators. Int J Intell Syst 1996;11:49-73.

31. Xu ZS. An overview of methods for determining OWA weights. Int J Intell Syst 2005;20:843-865.

32. Brenti F. Log-concave and unimodal sequences in algebra, combinatorics, and geometry: An update. In: Barcelo H, Kalai G, editors, Jerusalem Combinatorics '93, Contemporary Mathematics, vol. 178, 71-89. Providence RI: American Mathematical Society, 1994.

33. Yager RR, Filev DP. Parameterized and-like and or-like OWA operators. Int J Gen Syst 1994;22:297-316.

34. Filev DP, Yager RR. On the issue of obtaining OWA operator weights. Fuzzy Sets Syst 1998;94:157-169. 
35. Calvo T, De Baets B. Aggregation operators defined by $k$-order additive/maxitive fuzzy measures. Int J Uncertain Fuzziness Knowl-Based Syst 1998;06:533-550.

36. Ahn BS. On the properties of OWA operator weights functions with constant level of orness. IEEE Trans Fuzzy Syst 2006;14:511-515.

37. Sadiq R, Tesfamariam S. Probability density functions based weights for ordered weighted averaging (OWA) operators: An example of water quality indices. Eur J Oper Res 2007;182:1350-1368.

38. León T, Zuccarello P, Ayala G, de Ves E, Domingo J. Applying logistic regression to relevance feedback in image retrieval systems. Pattern Recognit 2007;40:2621-2632.

39. Lieb EH. Concavity properties and a generating function for Stirling numbers. J Comb Theory 1968;5:203-206.

40. Liu X. Models to determine parameterized ordered weighted averaging operators using optimization criteria. Inform Sci 2012;190:27-55.

41. Calvo T, Martín J, Mayor G, Torrens J. Balanced discrete fuzzy measures. Int J Uncertain Fuzziness Knowl-Based Syst 2000;08:665-676. 